\title{
Advanced Thyroid Carcinoma: Evolving Systemic Therapy Options
}

Presented by Lori J. Wirth, MD

\section{ABSTRACT}

Although multikinase inhibitors are established therapies in advanced thyroid cancers, treatment-related adverse events can lead to dose reductions and discontinuations, thereby limiting the effectiveness of these treatments. Gene-specific treatments have emerged as new standard-of-care therapies, and continue to demonstrate efficacy and improved tolerability, although genotyping is needed to identify patients that would benefit from these therapies and more education is needed in regard to the optimal use of these novel agents. Additionally, the emergence of acquired resistance has become a new problem in the field.

J Natl Compr Canc Netw 2021;19(5.5):658-661 doi: 10.6004/jnccn.2021.5018

Several multikinase inhibitors have established therapeutic benefit across advanced thyroid cancers. However, treatment-related adverse events (trAEs) are impactful in this patient population and may reduce the efficacy of these drugs, according to Lori J. Wirth, MD, Associate Professor, Harvard Medical School, and Medical Director, Head and Neck Oncology, Massachusetts General Hospital Cancer Center, at the NCCN 2021 Virtual Annual Conference. Gene-specific therapies are now demonstrating potent efficacy and improved tolerability in $R E T$ and NTRK-driven thyroid cancers, as well as in BRAF V600E-mutant anaplastic thyroid carcinomas (ATCs), and drugs that target these specific alterations have emerged as new standard-of-care therapies.

\section{The Array of Thyroid Cancers}

The classification of thyroid cancers is divided into 2 branch points: follicular-derived and parafollicular Ccell derived cancers. Follicular-derived thyroid cancers are seen most commonly; differentiated thyroid cancers (DTCs) fall under this category, and include follicular thyroid cancer, Hürthle cell carcinoma, poorly differentiated thyroid cancer (PDTC), and, the most common subtype, papillary thyroid cancer (PTC). ATC also falls under the classification of follicular-derived cancers.

Not commonly seen are medullary thyroid cancers (MTCs). These have a different natural history when compared with follicular-derived cancers, because they are derived from parafollicular C cells.

According Dr. Wirth, incredible advances have been made across all of these thyroid cancer types in oncology.
"Not Everyone With Thyroid Cancer Does Well" "I hear all the time that when my patients were first told they had thyroid cancer, their doctor told them they had the 'good cancer,' " said Dr. Wirth. "And then they end up seeing a medical oncologist and realize this is not a good cancer after all. So I don't like that phrase one bit." Not everyone with thyroid cancer does well, she reiterated, and when patients with DTC don't do well, they typically have recurrent (either locally unresectable and/or metastatic iodine-refractory) disease.

When patients have metastatic disease and they do experience response to radioactive iodine, their 10-year survival is $>90 \%$. "However, the patients who don't undergo treatment with radioactive iodine or [whose cancer] grows even if they do take up radioactive iodine have a 10 -year survival of only $10 \%$," she noted. "Those are the patients who are going to die of DTC."

Overall, MTC has an intermediate prognosis. "In general we are able to tease out the patients who will do poorly, based primarily on their calcitonin and CEA levels," she said. Calcitonin and CEA are both tumor markers for MTC. When patients have a good prognosis, they exhibit slowly increasing calcitonin and CEA doubling times. When the doubling time of either of these tumor markers is $>2$ years, those patients can have very good 10-year survival. However, when the doubling times are $<2$ years, overall 10 -year survival is poor, she said. ${ }^{2}$

According to Dr. Wirth, when it comes to ATC, almost no one does well. An analysis from the SEER database examining outcomes from $>1,500$ patients with ATC between 1986 and 2015 showed a median overall survival of only 4 months, with surgery showing the greatest impact on survival. ${ }^{3}$ 


\section{Approved Multikinase Inhibitors}

Approved multikinase inhibitors for iodine-refractory DTC include sorafenib and lenvatinib.

The DECISION trial was the first randomized phase III trial that investigated sorafenib compared with placebo in patients with progressive iodine-refractory DTC. ${ }^{4}$ The study investigators reported a significant progression-free survival (PFS) benefit of 5 months with sorafenib. "However, the overall response rate [ORR] was $12 \%$, not quite as high as we might have expected based on the phase II study," noted Dr. Wirth.

The SELECT trial investigated lenvatinib versus placebo in a similar patient population with progressive iodine-refractory DTC. ${ }^{5}$ This study showed a significant improvement in PFS of 14 months with lenvatinib compared with placebo and an ORR of $65 \%$.

These trials led to the FDA approvals of both sorafenib and lenvatinib for patients with iodine-refractory DTC.

Similarly, the FDA has approved 2 multikinase inhibitors for the treatment of MTC: vandetanib and cabozantinib. The ZETA trial demonstrated a PFS benefit of 11 months and an ORR of $45 \%$ with vandetanib compared with placebo in patients with MTC. ${ }^{6}$ "In this particular study, patients were not required to have disease progression at the time of study entry, so the PFS curve in the placebo group was $>19$ months," she said. "So this was a relatively indolent patient population."

In the EXAM trial, ${ }^{7}$ which investigated cabozantinib versus placebo, patients were required to have disease progression at the time of study entry. Study investigators found the placebo arm had a much shorter PFS of 4 months compared with 11 months for those in the cabozantinib arm, with an ORR of $28 \%$. Both vandetanib and cabozantinib are now FDA-approved for MTC.

Dr. Wirth pointed out that although the array of multikinase inhibition of these drugs provides them their activity in treating thyroid cancers, the same factor also contributes to the treatment-related toxicities associated with these therapies.

\section{trAEs With Multikinase Inhibitors}

"In terms of class effects of the multikinase inhibitors that are approved for treatment of advanced thyroid cancers, there are a number of trAEs that these drugs have in common," she said. Common class effects include rash (particularly palmar-plantar erythrodysesthesia), diarrhea, anorexia/weight loss, fatigue, and hypertension. More uncommon but potentially life-threatening AEs include QTc prolongation, bleeding/thromboembolism, and fistula. In general, these toxicities are managed with supportive care, as well as dose holds and dose reductions. In the aforementioned studies of advanced thyroid cancer, dose reductions were fairly common (occurring in $64 \%-79 \%$ of patients). ${ }^{4-7}$ Treatment discontinuation was also necessary in a subset of patients, ranging from $12 \%$ with vandetanib to $19 \%$ with sorafenib.

"But thyroid cancer is rich with druggable targets," said Dr. Wirth. Figure 1 outlines a number of potentially druggable targets across the array of follicular-derived thyroid cancers, including PTC, follicular thyroid cancer, Hürthle cell carcinoma, PDTC, and ATC. Researchers from Memorial Sloan Kettering examined $>10,000$ solid tumors profiled with next-generation sequencing for the MSK-IMPACT study and tabulated all of the potentially druggable mutations across the solid tumors. ${ }^{8}$

"Second only to gastrointestinal stromal tumors, thyroid cancers harbored the most common potentially druggable mixture of alterations in their assay, for a total of $61 \%$ of patients with thyroid cancer who had potentially druggable mutations or fusions," she said. "So targeted therapy is becoming a reality in thyroid cancer." Not only are druggable mutations relatively common in thyroid cancer, fusions are as well: across solid tumors studied in The Cancer Genome Atlas, PTC had the highest rate of recurrent oncogenic kinase fusions. ${ }^{9}$

\section{Gene-Specific Therapy in Thyroid Cancer}

In a phase II study of patients with iodine-refractory PTC and $B R A F$ V600E mutations, BRAF-directed therapy with dabrafenib \pm trametinib was well-tolerated and did display efficacy, but was not superior to first-line therapy with the multikinase inhibitor lenvatinib. ${ }^{10}$ "However, interestingly, this combination [dabrafenib + trametinib] seems to work pretty well in BRAF V600E ATC," she noted.

Approximately $30 \%$ to $40 \%$ of patients with ATC harbor BRAF V600E mutations. In a cohort of 16 patients with BRAF V600E ATC enrolled in a phase II basket trial, treatment with dabrafenib + trametinib led to an ORR of $63 \%$ and estimated overall survival of $80 \%$ at 12 months. ${ }^{11}$ "For ATC, an ORR of $63 \%$ is really remarkable," she noted. "Another thing that was remarkable about these results was that the responses were quite durable." Based on this basket trial experience in only 16 patients, dabrafenib + trametinib was FDA-approved for the treatment of BRAF-mutant ATC.

RET mutations are noted in approximately $70 \%$ of all MTCs, with RET M918T being the most common somatic mutation and RET C634 the most common hereditary mutation. ${ }^{12,13}$ In terms of RET fusion-positive thyroid cancer, RET fusions are observed in $<10 \%$ of PTCs overall, but are seen more frequently in pediatric and young adult cancers, occurring in approximately $30 \%$ of these malignancies. CCDC6-RET (RET/PTC1) and NCOA4-RET (RET/PTC3) are the most common fusions, but at least 25 $5^{\prime}$ fusion partners have now been identified in thyroid cancer. 

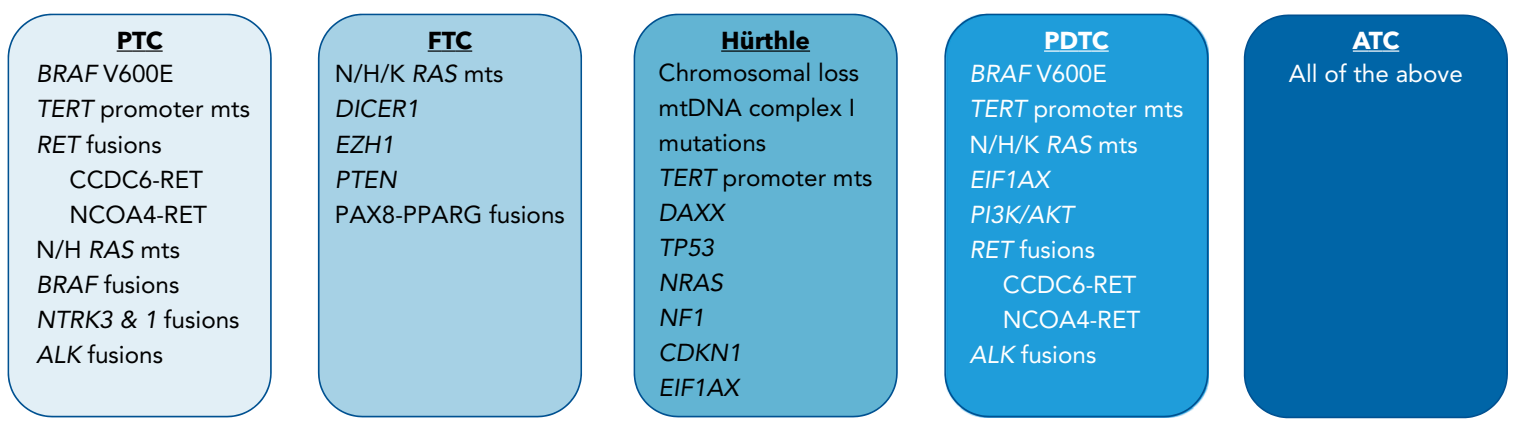

Figure 1. Thyroid cancer rich with druggable targets.

Abbreviations: ATC, anaplastic thyroid carcinoma; FTC, follicular thyroid carcinoma; mts, mutations; PDTC, poorly differentiated thyroid cancer; PTC, papillary thyroid cancer.

Data from refs $21-25$

Two new highly potent and specific RET inhibitors, selpercatinib and pralsetinib, have now completed their first in-human trials. "These agents were designed to have as little activity against KDR/VEGFR-2 as possible, because we think that most of the toxicities of VEGFR multikinase inhibitors are due to inhibition of KDR/ VEGFR-2," she explained. "The idea is that the efficacy of the other multikinase inhibitors may be limited by insufficient RET inhibition due to dose-limiting toxicities, and that limits the RET blockade that you can get with multikinase inhibitors."

In the open-label phase I/II LIBRETTO-001 trial of selpercatinib in RET-driven non-small cell lung cancer (NSCLC), thyroid cancers, and other RET-driven cancers, ${ }^{14,15}$ patients with $R E T$-altered thyroid cancers treated with selpercatinib demonstrated responses across all $R E T$ mutations, including RET V804, the "gatekeeper" resistance mutation. Of the 3 thyroid cohorts in the trial, the primary analysis subgroup consisted of patients with $R E T$ mutated MTC previously treated with vandetanib \pm cabozantinib. In these patients, the ORR was $69 \%$, with $9 \%$ of patients demonstrating complete responses and 60\% showing partial responses. Responses were seen across all RET mutations studied, including RET V804. Overall, selpercatinib was well-tolerated and responses were durable (median duration of response and median PFS were not yet reached in the primary analysis cohort at the time of publication). In the 19 patients with previously treated RET fusion-positive thyroid cancer, the ORR was $79 \%$.

"It was on the basis of these results in the thyroid cancer cohorts, as well as in the lung cancer cohorts, that the FDA approved selpercatinib for RET-altered lung and thyroid cancers in a line-agnostic approval," said Dr. Wirth. ${ }^{15}$

Pralsetinib was investigated in the similarly designed phase I/II ARROW trial that enrolled patients with RETdriven NSCLC and RET-driven thyroid cancers (previously treated and untreated). ${ }^{16}$ "Again, responses are being observed across all of the RET mutations enrolled, including those with the RET V804 gatekeeper resistance mutation," reported Dr. Wirth. Responses were also durable with pralsetinib: both median duration of response and median PFS had not yet been reached at the time of publication. Overall, the treatment was also well tolerated. Based on data from the ARROW trial, the FDA has approved pralsetinib for patients with $R E T$-driven advanced thyroid cancers and NSCLC.

\section{Targeting NTRK Fusions}

NTRK $1 / 3$ fusions present in approximately $2 \%$ of PTCs and, just like RET fusions, are more common in pediatric and young adult patients with thyroid cancer. Larotrectinib has been studied across multiple tumor types in patients that harbor NTRK fusions, including in those with NTRK fusion-positive DTC and ATC. ${ }^{17}$ Thyroid cancer was the second most common adult tumor type enrolled in the larotrectinib trials. Of 21 patients with NTRK fusion-positive iodine-refractory DTC, $90 \%$ responded to larotrectinib. "Response rates in ATC were perhaps a little bit disappointing at $29 \%$, but those response rates for ATC are really pretty good for that particular diagnosis," she noted. "So on the basis of the larotrectinib experience, larotrectinib was the first drug in oncology to receive a tumor-agnostic approval by the FDA."18

Although gene-specific therapy has led to great advances in the treatment of advanced thyroid cancer, one particular challenge in the field is identifying patients in need of systemic therapy that harbor these alterations, she added.

\section{Looking Forward}

According to Dr. Wirth, the emergence of acquired resistance is a new problem in the field of thyroid cancer. Acquired resistance on selpercatinib/pralsetinib has now been described. ${ }^{19,20}$ In $>20$ patients, acquired RET G810 kinase solvent front mutations accounted for the development of resistance in 10\% and acquired MET amplification has been described in approximately $15 \%$ of 
patients who experienced disease progression on either selpercatinib or pralsetinib.

A phase I/II trial will evaluate TPX-0046, a nextgeneration $R E T$-specific inhibitor (ClinicalTrials.gov identifier: NCT04161391). "This is a novel, potent RET/SRC inhibitor that was also designed to overcome the acquired solvent front mutation at G810," she said. "This trial is open and enrolling at multiple sites. I want to highlight the fact that we're already studying our nextgeneration kinase inhibitors in clinical trials."

Disclosures: Dr. Wirth has disclosed serving as a scientific advisor for Bayer HealthCare, Blueprint Medicines, Cue Biopharma, Eisai Inc., Eli Lilly and Company, Genentech, Inc., Loxo Oncology, Inc., and Merck \& Co., Inc.

Correspondence: Lori J. Wirth, MD, Massachusetts General Hospital Cancer Center, 55 Fruit Street, Boston, MA 02114

Email: Iwirth@mgh.harvard.edu

\section{References}

1. Durante C, Haddy N, Baudin E, et al. Long-term outcome of 444 patients with distant metastases from papillary and follicular thyroid carcinoma: benefits and limits of radioiodine therapy. J Clin Endocrinol Metab 2006;91:2892-2899.

2. Barbet J, Campion L, Kraeber-Bodere F, et al. Prognostic impact of serum calcitonin and carcinoembryonic antigen doubling-times in patients with medullary thyroid carcinoma. J Clin Endocrinol Metab 2005;90:6077-6784

3. Lin $\mathrm{B}, \mathrm{MaH}, \mathrm{Ma} \mathrm{M}$, et al. The incidence and survival analysis for anaplastic thyroid cancer: a SEER database analysis. Am J Transl Res 2019;11:5888-5896.

4. Brose MS, Nutting CM, Jarzab B, et al. Sorafenib in radioactive iodine-refractory, locally advanced or metastatic differentiated thyroid cancer: a randomised, double-blind, phase 3 trial. Lancet 2014384:319-328.

5. Schlumberger $M$, Tahara M, Wirth LJ, et al. Lenvatinib versus placebo in radioiodine-refractory thyroid cancer. N Engl J Med 2015;372:621-630.

6. Wells SA, Robinson BG, Gagel RF, et al. Vandetanib in patients with locally advanced or metastatic medullary thyroid cancer: a randomized, double-blind phase III trial. J Clin Oncol 2012;30:134-141.

7. Elisei R, Schlumberger MJ, Muller SP, et al. Cabozantinib in progressive medullary thyroid cancer. J Clin Oncol 2013;31:3639-3646.

8. Zehir A, Benayed R, Shah RH, et al. Mutational landscape of metastatic cancer revealed from prospective clinical sequencing of 10,000 patients. Nat Med 2017;23:703-713.

9. Stransky N, Cerami E, Schalm S, et al. The landscape of kinase fusions in cancer. Nat Commun 2014;5:4846.

10. Shah $M H$, Wei $L$, Wirth LJ, et al. Results of randomized phase II trial of dabrafenib versus dabrafenib plus trametinib in BRAF-mutated papillary thyroid carcinoma [abstract]. J Clin Oncol 2017;15(Suppl):Abstract 6022.

11. Subbiah V, Kreitman RJ, Wainberg ZA, et al. Dabrafenib and trametinib treatment in patients with locally advanced or metastatic BRAF V600-mutant anaplastic thyroid cancer. J Clin Oncol 2018;36:7-13.

12. Ciampi R, Romei C, Ramone T, et al. Genetic landscape of somatic mutations in a large cohort of sporadic medullary thyroid carcinomas studied by next-generation targeted sequencing. iScience 2019;20:324-336.

13. Mulligan LM. RET revisited: expanding the oncogenic portfolio. Nat Rev Cancer 2014;14:173-186.
14. Wirth LJ, Sherman E, Robinson B, et al. Efficacy of selpercatinib in RET-altered thyroid cancers. N Engl J Med 2020;383:825-835.

15. Drilon A, Oxnard GR, Tan DSW, et al. Efficacy of selpercatinib in RET fusion-positive non-small-cell lung cancer. N Engl J Med 2020;383:813-824

16. Hu M, Subbiah $V$, Wirth $L J$, et al. Results from the registrational phase $1 / \mid$ ARROW trial of pralsetinib (BLU-667) in patients (pts) with advanced RET mutation-positive medullary thyroid cancer (RET+ MTC) [abstract]. Ann Oncol 2020;31(Suppl 4):Abstract 19130.

17. Hong DS, DuBois SG, Kummar $S$, et al. Larotrectinib in patients with TRK fusion-positive solid tumours: a pooled analysis of three phase 1/2 clinical trials. Lancet Oncol 2020;21:531-540.

18. Cabanillas ME, Drilon A, Farago AF, et al. Larotrectinib treatment of advanced TRK fusion thyroid cancer [abstract]. Ann Oncol 2020;31(Suppl 4):Abstract 1916P.

19. Solomon BJ, Tan L, Lin JJ, et al. RET solvent front mutations mediate ac quired resistance to selective RET inhibition in RET-driven malignancies. J Thorac Oncol 2020;15:541-549.

20. Lin JJ, Liu SV, McCoach CE, et al. Mechanisms of resistance to selective RET tyrosine kinase inhibitors in RET fusion-positive non-small-cell lung cancer. Ann Oncol 2020;31:1725-1733.

21. The Cancer Genome Atlas Research Network. Integrated genomic characterization of papillary thyroid carcinoma. Cell 2014;159:676-690.

22. Yoo SK, Lee S, Kim SJ, et al. Comprehensive analysis of the transcriptional and mutational landscape of follicular and papillary thyroid cancers. PLoS Genet 2016;12:e1006239.

23. Gopal RK, Kübler K, Calvo SE, et al. Widespread chromosomal losses and mitochondrial DNA alterations as genetic drivers in Hürthle cell carcinoma. Cancer Cell 2018;34:242-255.e5.

24. Ganly I, Makarov V, Deraje S, et al. Integrated genomic analysis of Hürthle cell cancer reveals oncogenic drivers, recurrent mitochondrial mutations, and unique chromosomal landscapes. Cancer Cell 2018;34:256-270.e5.

25. Landa I, Ibrahimpasic T, Boucai L, et al. Genomic and transcriptomic hallmarks of poorly differentiated and anaplastic thyroid cancers. J Clin Invest 2016;126:1052-1066. 